\title{
ARTICLE Selective delipidation of Mycobacterium bovis BCG enables direct pulmonary vaccination and enhances protection against Mycobacterium tuberculosis
}

\author{
Juan I. Moliva ${ }^{1,2}$, Austin P. Hossfeld ${ }^{2}$, Sabeen Sidiki ${ }^{2}$, Cynthia H. Canan ${ }^{2}$, Varun Dwivedi ${ }^{1}$, Gillian Beamer ${ }^{3}$, Joanne Turner ${ }^{1,2}$ and $^{2}$ \\ Jordi B. Torrelles ${ }^{1,2}$
}

Mycobacterium tuberculosis (M.tb), the causative agent of tuberculosis (TB), is the leading killer due to an infectious organism. Mycobacterium bovis bacillus Calmette-Guérin (BCG) is the only vaccine approved against TB, however, its efficacy against pulmonary TB is poor. While BCG is currently inoculated intradermally, the natural route of M.tb infection is through the lung. Excessive lung pathology caused by pulmonary inoculation of BCG has prevented the use of this immunization route. Here, we show that selective chemical treatment of BCG with petroleum ether removes inflammatory lipids from the bacterial surface while keeping BCG viable. Pulmonary vaccination using this modified BCG attenuated inflammatory responses, prevented immunopathology of the lung, and significantly increased protection against M.tb infection in mice. We further directly linked IL$17 \mathrm{~A}$ as the responsible contributor of improved immunity against M.tb infection. These results provide evidence that selective removal of cytotoxic lipids from the BCG surface attenuates inflammation and offers a safer and superior vaccine against TB causing less damage post-infectious challenge with M.tb.

Mucosal Immunology (2019) 12:805-815; https://doi.org/10.1038/s41385-019-0148-2

\section{INTRODUCTION}

Mycobacterium tuberculosis (M.tb) the causative agent of tuberculosis (TB) continues to cause significant morbidity and mortality around the world, and the rise of extensive-, extreme-, and total-drug resistant M.tb has endangered eradication efforts. ${ }^{1}$ The currently licensed vaccine against TB, Mycobacterium bovis Bacille Calmette et Guérin (BCG), is only partially effective against pulmonary TB (PTB) despite it being highly efficacious against other forms of mycobacterial disease such as TB meningitis and miliary TB. ${ }^{2,3}$ One potential explanation for this discrepancy may lie in the route of immunization as BCG is administered intradermally, whereas $M . t b$ is a natural airborne pathogen. ${ }^{4}$ This noted disparity may explain why BCG fails to confer optimum anti-mycobacterial immunity in the lung. To circumvent this issue research has shifted toward direct pulmonary vaccination with BCG. ${ }^{5-7}$ Evidence suggests that BCG is too pathogenic to be utilized as a direct pulmonary vaccine as it can induce significant pulmonary immunopathology. ${ }^{4,8,9}$ For this reason, no human clinical trial has yet been implemented to evaluate the efficacy of pulmonary BCG vaccination against M.tb.

The primary reason behind the immunopathology caused by mycobacteria to the lung is attributed to potent inflammatory and immunomodulatory lipids such as trehalose dimycolate (TDM), ${ }^{10,11}$ $\mathrm{di}-$ and tri-acylglycerols (DAG/sTAGs), ${ }^{12}$ phthiocerol dimycocerosates (PDIMs), ${ }^{13}$ phenolic glycolipids (PGLs), ${ }^{14,15}$ among others, present on the BCG cell wall. These lipids induce rapid and robust innate immune responses that lead to tissue inflammation and damage $^{16}$ or are used by mycobacteria to subvert host immunity. ${ }^{13,17-20}$ However, recombinant BCG strains deficient in specific lipids or a combination of these lipids are weak inducers of immune responses that protect mice against $M . t b .^{21}$ Thus, it is suggested that complete absence of the cell wall lipids is detrimental to the generation of immunity, but their presence is responsible for lung pathology.

In this study, we recapitulated and extended previous studies by Silva et al. ${ }^{11}$ We demonstrate that treatment of BCG with the organic solvent petroleum ether (PE) selectively extracts many of the inflammatory lipids, including TDM, PGL, mycoside $B$ $(\mathrm{MycB})$, and some triacylglycerols (TAGs) and PDIMs without affecting the viability of BCG. Using primary human macrophages, we demonstrate qualitative and quantitative lowered innate inflammatory responses to delipidated BCG (dBCG) compared to conventional BCG. This observation led us to hypothesize that delipidation of BCG ( $\mathrm{dBCG}$ ) could be used as an effective pulmonary vaccine as it would bypass the pathology associated with this route of vaccination while retaining the efficacy associated with direct inoculation of the lung. We show that direct inoculation of the lung with $\mathrm{dBCG}$ significantly reduced the inflammatory status of the lung in vaccinated mice compared to conventional BCG. Furthermore, vaccination with $\mathrm{dBCG}$ conferred superior protection relative to conventional BCG against challenge with M.tb. Protection against M.tb infection by $\mathrm{dBCG}$ vaccination was associated with

\footnotetext{
${ }^{1}$ Texas Biomedical Research Institute, San Antonio, TX 78227, USA; ${ }^{2}$ Department of Microbial Infection and Immunity, Wexner College of Medicine, The Ohio State University, Columbus, OH 43210, USA and ${ }^{3}$ Department of Infectious Diseases and Global Health, Cummings School of Veterinary Medicine, Tufts University, North Grafton, MA 01536, USA Correspondence: Joanne Turner (JoanneTurner@txbiomed.org) or Jordi B. Torrelles (JTorrelles@txbiomed.org)
}

Received: 31 August 2018 Revised: 2 January 2019 Accepted: 27 January 2019

Published online: 18 February 2019 



Fig. 1 Petroleum ether extracts TDM, PGL, MycB, PDIMs, and TAGs without affecting the viability of BCG. To assess nature and percentage of the lipids remaining on the BCG cell wall following extraction with PE (PE BCG extract), PE treated BCG was subjected to C:M (2:1, v/v) extraction for $12 \mathrm{~h}$ at $37^{\circ} \mathrm{C}$ (C:M BCG extract) ( $n=3$; TLC, C:M, 95:5 v/v). Representative TLC images of PE BCG extracts in triplicate. BCG total lipid (BCG TL) extracts were included as a reference. a TDM, MycB, and PGL are highly extractable by PE. b Some PDIMs and TAGs are extracted from BCG by PE $(n=3$; TLC, petroleum ether/acetone, 96:4 v/v). c PIMs are not extractable by PE $(n=3$, TLC, chloroform/acetic acid/methanol/ water, 40:25:3:6 v/v/v/v). d Densitometry analysis of all lipids plotted as percent extracted following treatment with the corresponding solvents, (PE BCG extracts, grey bars), (C:M BCG extracts, black bars), $(n=3)$. e The viability of BCG treated with PBS, PE or C:M (2:1, v/v) assessed by plating serial dilutions $(n=6)$. Representative experiment shown, each experiment performed at least three times, mean \pm SEM; unpaired Student's $t$-test, ${ }^{* *} p<0.001 ;$ ns: not significant, nd: no detected

increased numbers of effector and central memory $\mathrm{T}$ cell populations in the lung, and with increased $\mathrm{CD}^{+} 9^{+}$and IL-17 $\mathrm{A}^{+}$, but not IFNY ${ }^{+}$, T cell responses. Depletion of IL-17A throughout the vaccination period abrogated protection in the lung of $\mathrm{dBCG}$-vaccinated mice. Altogether, our results provide proof of concept that $\mathrm{dBCG}$ can be easily adapted into a pulmonary vaccine with few safety concerns and enhanced efficacy against M.tb.

\section{RESULTS}

PE treatment extracts non-polar lipids from BCG without affecting bacillus viability, attenuating innate inflammatory responses Lipid extraction from PE treated BCG was assessed by thin layer chromatography (TLC). Three independent PE extractions are shown (PE BCG Extract). BCG total lipids were included on TLCs as controls. To assess the amount and the makeup of the remaining lipids on BCG after PE extraction, bacteria were further extracted with chloroform:methanol (2:1, v/v) (C:M BCG Extract). As described, ${ }^{11,22-24}$ TDM was highly extractable using PE resulting in a consistent removal of 70\%-90\% TDM from BCG (Fig. 1a, d). Due to the hydrophobic nature of $P E$, we also observed significant extraction of $\mathrm{PGL}$ and $\mathrm{MycB}$, both important virulence factors associated with the mycobacterial cell wall (Fig. 1a, d). Additionally, some PDIMs and TAGs were partially extractable (Fig. 1b, d). As expected, PE was unable to remove phospholipids such as phosphatidyl-myo-inositol mannosides (PIMs) from the BCG cell wall, and PIMs were only extractable after C:M extraction (Fig. 1e, d). We further confirmed that large chain carbohydrates such as mannose-capped lipoarabinomannan (ManLAM) were not directly or indirectly extractable with PE (see Sup. Fig. S1A) and remained on the PE treated BCG cell wall (see Sup. Fig. S1B).

To confirm that lipid extraction by $\mathrm{PE}$ was not lethal or inhibitory for optimal growth of BCG, we plated PBS-treated, PEtreated, or PE + C:M-treated BCG. As previously described, ${ }^{11,22}$ no significant differences in growth between PBS and PE treated BCG was observed, whereas PE + C:M-treated BCG did not grow (Fig. 1e). We confirmed that dBCG generated a single cell suspension of bacteria (see Sup. Fig. S2). Therefore, PE extracted a significant portion of cytotoxic, non-polar molecules from the BCG cell wall with high reproducibility, and without affecting BCG viability.

The consequence of removing non-polar lipids on the viability and innate inflammatory responses of BCG was first determined in vitro in primary human macrophages. Despite equal inoculums (Fig. S3A), BCG and dBCG had an average uptake of $9.66 \pm 5.51$ and $3.00 \pm 1.32(\mathrm{M} \pm \mathrm{SD})$, respectively, suggesting that non-polar lipids may directly impact the interaction between BCG and human macrophages. Growth of $d B C G$ in 

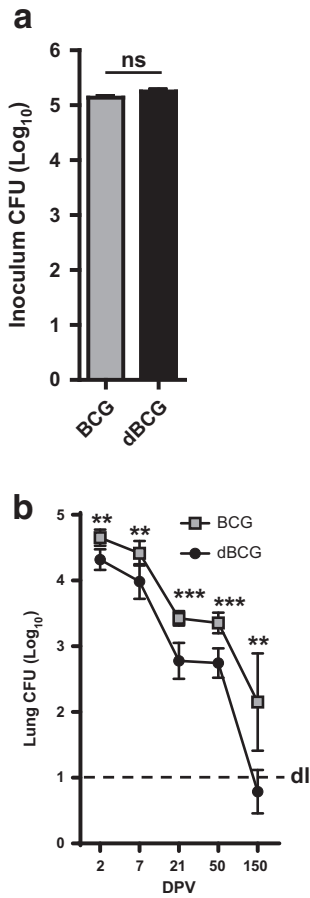

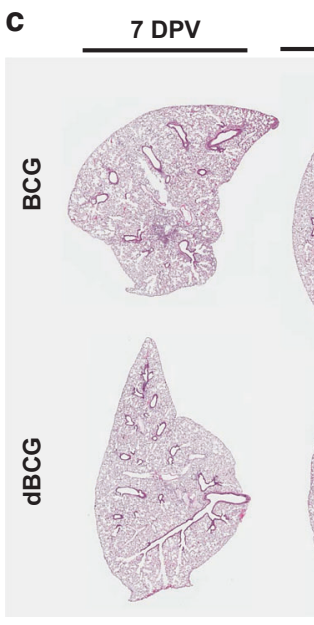

21 DPV

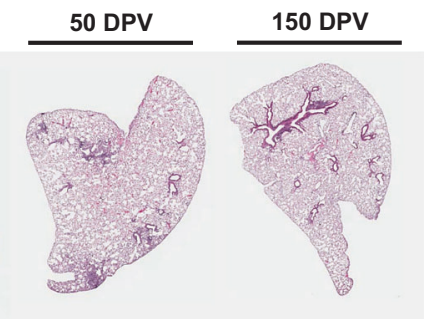

d
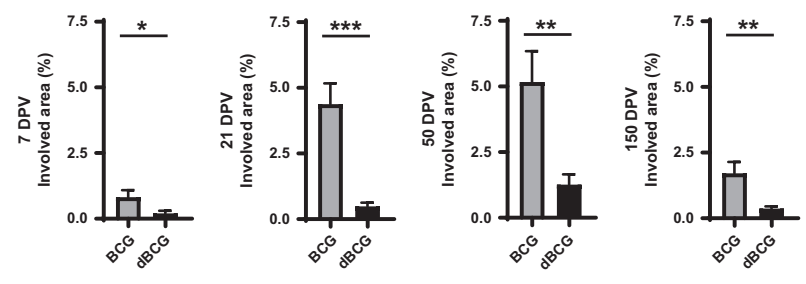

Fig. 2 Delipidated BCG is quickly eliminated from the lung and is associated with reduced pathology of the lung. C57BL/6 mice were intranasally inoculated with $5 \times 10^{5}$ viable BCG (grey bar or grey circle) or dBCG (black bar or black circle) bacilli. Mice were sacrificed at 2, 7, 21, 50 , and 150 DPV to assess BCG bacterial burden in the lung. a Inoculum (i.e., the number of bacteria delivered into the lung) used to vaccinate mice showed no significant differences between the two groups. Representative experiment of $n=2$, mean \pm SEM; unpaired Student's $t$-test; ns: not significant. (b) BCG CFUs in the lung assessed at 2, 7, 21, 50, and 150 DPV. Pooled results from $n=2$ with 4-5 mice/group per timepoint, mean and error $\pm 95 \% \mathrm{Cl}$; unpaired Student's $t$-test, ${ }^{* *} p<0.01,{ }^{* *} p<0.001$. c Representative images of lung tissue at 7, 21, 50, and 150 DPV. $\mathbf{d}$ Areas of cell aggregation and infiltration (inflammation) in BCG (grey bars) or dBCG (black bars) vaccinated mice were quantified using Aperio Imagescope by calculating the area of inflammatory foci (i.e., involvement) divided by the total area of the lung. Representative images at a final magnification of $\times 20$. Representative experiment from $n=2$ with $4-5$ mice/group, mean \pm SEM; unpaired Student's $t$-test, ${ }^{*} p<0.05$, ${ }^{* *} p<0.01,{ }^{* *} p<0.001$

macrophages was significantly reduced when compared to conventional BCG (Fig. S3B), and dBCG inoculated macrophages released significantly less TNF, IL-1 $\beta$, IL-6, and IL-10, but not IL12 p40 (Fig. S3C, D), supporting previous findings using delipidated M.tb, where both cytokine reduction in spite of better bacterial killing was also observed. ${ }^{22}$ Moreover, lower levels of secreted lactose dehydrogenase (LDH) were detected in $d B C G$ inoculated macrophages (Fig. S3E). Images of dBCG inoculated macrophages captured at $120 \mathrm{~h}$ post infection corroborated these findings (Fig. S3F).

dBCG attenuates pulmonary inflammation in vivo following intranasal vaccination and alters cell populations of the lung Our in vitro data indicate that removal of non-polar lipids reduced inflammatory cytokine secretion (Fig. S3) and therefore we next sought to evaluate the potential of $\mathrm{dBCG}$ as a pulmonary vaccine. C57BL/6 mice were inoculated intranasally with $5.0 \times 10^{5}$ BCG or $\mathrm{dBCG}$ bacilli. Despite equal inoculums (Fig. 2a), the ability of dBCG to persist in the lung was significantly reduced by day 2 post vaccination (DPV), a trend that continued for up to 150 days, with a continuous decrease in bacteria burden (Fig. 2b). By 150 DPV, CFUs in the lung of dBCG-vaccinated mice were below accurate detection levels, whereas BCG-vaccinated mice had higher CFUs in the lung (Fig. 2b). The levels of TNF and IL-6 in the lungs of dBCGvaccinated mice were significantly decreased as early as 7 DPV and the levels of IL-1 $\beta, I L-10$, and IFN $\gamma$ displayed the same trend (Sup. Fig. S4). By 50 DPV, all measured cytokines except for IL$12 \mathrm{p} 40$ were significantly decreased in the dBCG group. Altogether, the data suggest that chemical removal of non-polar lipids from the BCG cell wall significantly impacts its ability to persist in vivo and diminishes inflammatory responses within the lung. To corroborate this, we analyzed the immunopathology of the lung in BCG- or dBCG-vaccinated mice. Lung inflammation was determined by quantifying the size of the inflammatory foci over the total area of the lung lobe. The lung of BCG-vaccinated mice had more extensive areas of inflammation and cellular infiltration compared to dBCG-vaccinated mice at 7 DPV (Fig. 2c, d). This same trend was observed at 21 and 50 DPV with larger foci visible in BCG-vaccinated mice compared to dBCG-vaccinated animals. By $150 \mathrm{DPV}$, inflammation in the lung of BCG-vaccinated mice had decreased but remained significantly higher compared to dBCGvaccinated mice. Together, the data suggest that $d B C G$ induces immunopathology to a lesser degree than BCG when it is administered directly into the lung, and thus could be a safe pulmonary vaccine.

We assessed immune cell kinetics in the lung of vaccinated mice across 50 days (for gating scheme see Sup. Fig. S5A). Early post vaccination we found significantly reduced percentages of monocytes and neutrophils in the lung of mice vaccinated with dBCG relative to BCG (Sup. Fig. S6A, B). We observed no significant difference in the percentages of alveolar macrophages (AMs) and dendritic cells (DCs) in the lung between groups (Sup. Fig. S6C, D), however, the percentage of AMs decreased significantly from 7 to 21 and 50 DPV. The percentage of DCs increased over time in both groups relative to naive mice and were found to be significantly elevated in the lung of $\mathrm{dBCG}$ vaccinated mice at $50 \mathrm{DPV}$. We found that $B$ cell numbers increased significantly across time in both vaccination groups (Sup. Fig. S6E). The number of NK cells decreased across time but was significantly increased in BCGvaccinated mice relative to $\mathrm{dBCG}$-vaccinated mice at 50 DPV (Sup. 
CD4 ${ }^{+} \mathrm{T}$ cells

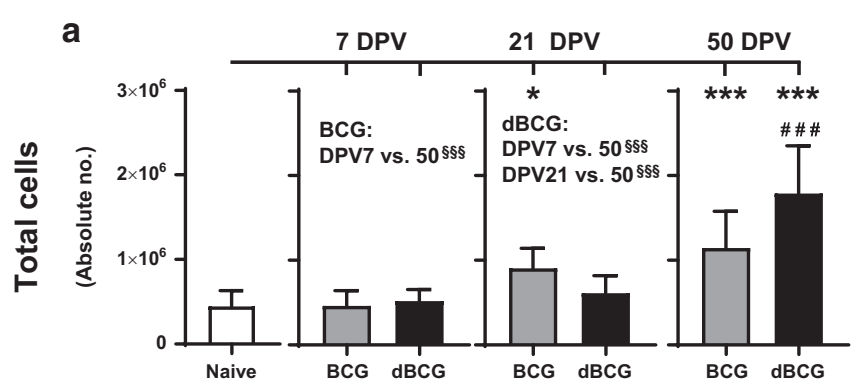

C

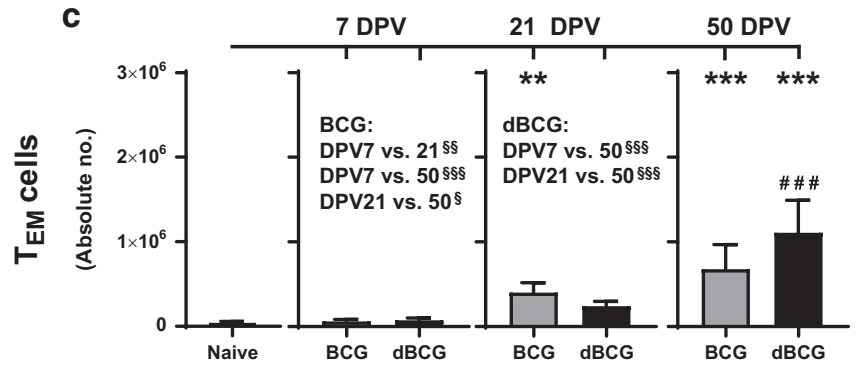

e

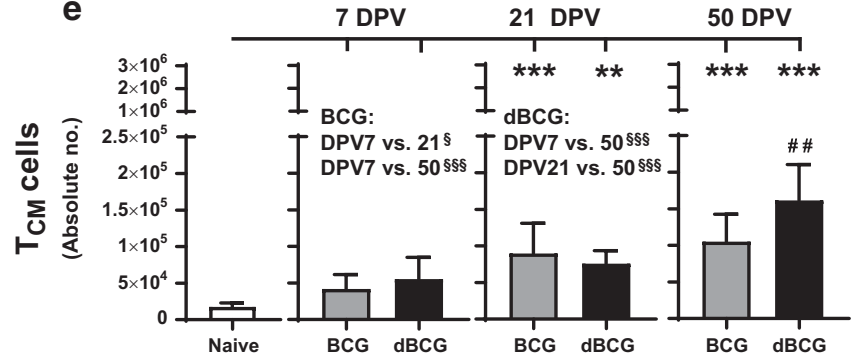

$\mathrm{CD}^{+} \mathrm{T}$ cells

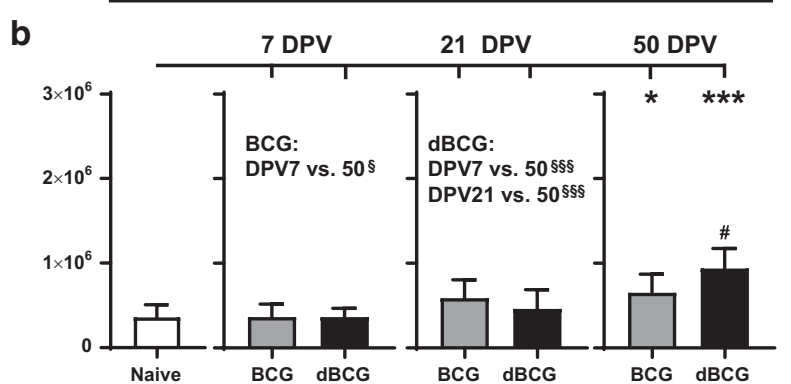

d

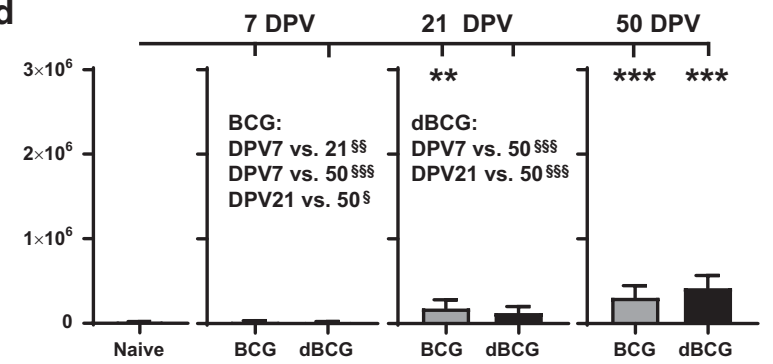

f

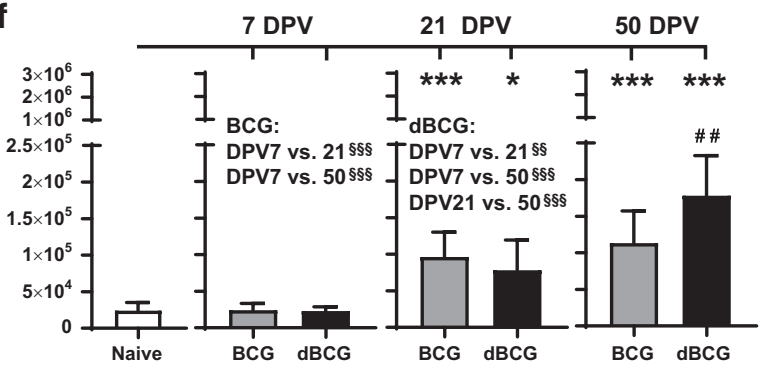

Fig. 3 Pulmonary vaccination with delipidated BCG increases memory and effector $T$ cell responses in the lung. C57BL/6 mice were intranasally inoculated with $5 \times 10^{5}$ viable BCG (grey squares) or dBCG (black circles) bacilli. A group of naïve mice (white circles) was included as a control. Mice were sacrificed at 7, 21, and 50 DPV to characterize memory T cell responses in the lung. Total number of (a) CD4 ${ }^{+}$and (b) $\mathrm{CD}^{+} \mathrm{T}$ cells in the lung across time. The proportion of $(\mathbf{c}) \mathrm{CD}^{+}$or $(\mathbf{d}) \mathrm{CD}^{+}$effector $\mathrm{T}$ cells $\left(\mathrm{CD} 62 \mathrm{~L}^{-} \mathrm{CD} 44^{+}\right)$increased across time and reached statistical significance in dBCG vaccinated mice at 50 DPV. The proportion of (e) $\mathrm{CD}^{+}$or $(\mathbf{f}) \mathrm{CD}^{+}$memory $^{\mathrm{T}}$ cells $\left(\mathrm{CD} 62 \mathrm{~L}^{+} \mathrm{CD} 44^{+}\right.$) also increased over time and peaked at 50 DPV in dBCG vaccinated mice. Pooled experiment from $n=2$ with 4-5 mice/group, mean \pm SEM; One-way ANOVA with the Holm-Sidak's multiple comparison test. The bar on the top compares each group across time with the control group (naive) $\left({ }^{*} p<0.05 ;{ }^{* *} p<0.01 ;{ }^{* *} p<0.001\right)$. Comparisons among BCG or dBCG groups across time points is represented by the symbol $\S\left({ }^{\S} p<0.05 ;{ }^{\S} p<0.01 ;{ }^{\S \S} p<0.001\right)$. Comparisons between groups at the same time point are represented by the symbol $\sharp(\sharp p<0.05 ; \sharp \sharp p<$ $0.01 ; \# \# \#<0.001)$. Where not stated, it was not significant. For clarity, statistically significant comparisons across time points within the same group (BCG or dBCG) has been written within the figure (i.e. BCG: DPV7 vs. $21{ }^{\S \S} p<0.01$ ). DPV day post vaccination

Fig. S6F), while the percentage of $\gamma \delta^{+} T$ cells was not affected by either formulation, despite overall significant increases across time (Sup. Fig. S6G).

Pulmonary vaccination with $\mathrm{dBCG}$ increases memory $\mathrm{T}$ cell populations in the lung

BCG is the only licensed vaccine that is thought to primarily mediate protection via the action of memory $\mathrm{CD} 4^{+}$and to a lesser extent $\mathrm{CD} 8^{+} \mathrm{T}$ cells ${ }^{25}$ that respond rapidly upon infection with $M$. $t b .{ }^{26}$ For these reasons, we next focused on the proportions of $\mathrm{CD}^{+}$and $\mathrm{CD}^{+}{ }^{+} \mathrm{T}$ cells expressing markers associated with effector memory $\left(\mathrm{T}_{\mathrm{EM}}-\mathrm{CD} 62 \mathrm{~L}^{-} \mathrm{CD} 44^{+}\right)$and central memory $\left(\mathrm{T}_{\mathrm{CM}}-\right.$ $\mathrm{CD}_{22} \mathrm{~L}^{+} \mathrm{CD}_{4}{ }^{+}$) in the lung of BCG-vaccinated and $\mathrm{dBCG}-$ vaccinated mice. The proportion of $\mathrm{CD}^{+}$and $\mathrm{CD}^{+}{ }^{+} \mathrm{T}$ cells increased across time following vaccination with BCG and $\mathrm{dBCG}$ vaccinated mice, but mice vaccinated with $\mathrm{dBCG}$ had significantly increased numbers of $\mathrm{CD}^{+}$and $\mathrm{CD}^{+} \mathrm{T}$ cells in the lung at 50 DPV (Fig. 3a, b and Sup. Fig. S5B). Proportions of $\mathrm{T}_{E M} \mathrm{CD}^{+}$and $\mathrm{CD}^{+}$cells in the lung increased across time in BCG and $\mathrm{dBCG}$ vaccinated mice, with dBCG having significantly more $C D 4^{+} \mathrm{T}_{\mathrm{EM}}$ at 50 DPV (Fig. 3c, d). $T_{E M}$ cells are believed to possess short-lived immunological memory, while tissue-resident $T_{C M}$ cells, though far fewer than $T_{E M}$ in tissue, are longer-lived. ${ }^{27}$ We found that $\mathrm{dBCG}$ induced greater proportions of $\mathrm{CD}^{+}$and $\mathrm{CD}^{+} \mathrm{T}_{\mathrm{CM}}$ cells in the lung compared to BCG vaccination (Fig. 3e, f).

M.tb challenge after pulmonary vaccination with $\mathrm{dBCG}$ attenuates pulmonary immunopathology and affords superior protection relative to conventional $B C G$

To replace the current $T B$ vaccine, the new candidate must be more efficacious at reducing M.tb bacterial burden and preventing TB pathology. Mice were randomized into vehicle (saline), BCG, or dBCG groups and vaccinated with $5 \times 10^{5}$ bacteria via intranasal inoculation of the lung. 50 days post vaccination, all groups were infected simultaneously with a low dose aerosol of M.tb. ${ }^{28}$ Bacterial burden was assessed in the lung, spleen, liver, and mediastinal lymph node (MLN) at 21,60, and 150-days postinfection (DPI). We observed a significant decrease in lung bacterial burden at every time point in mice vaccinated with $\mathrm{dBCG}$, compared to vehicle control and conventional BCG (Fig. 4a). We also observed a significant delay in colonization of the spleen (Fig. 4b), liver (Fig. 4c), and MLN (Fig. 4d) in dBCG-vaccinated mice. 

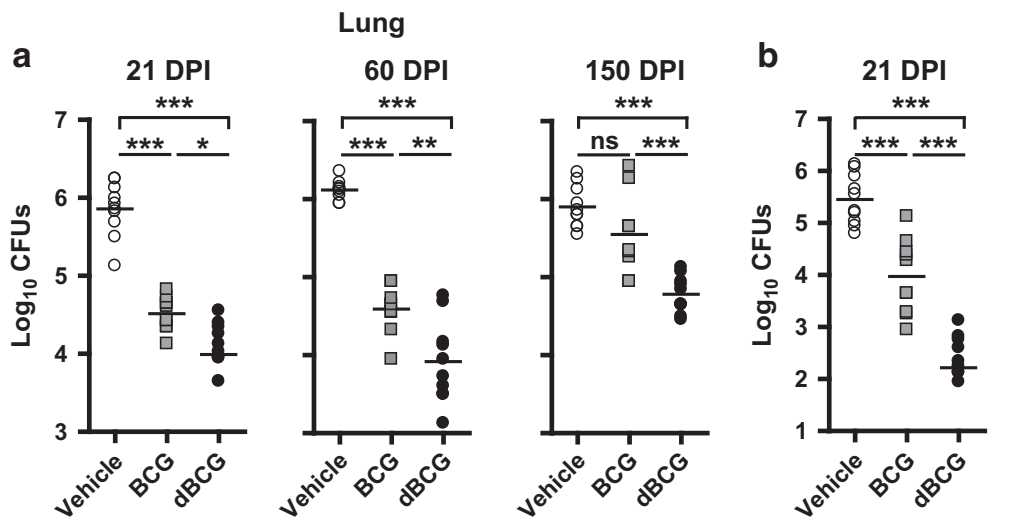

\section{Spleen}
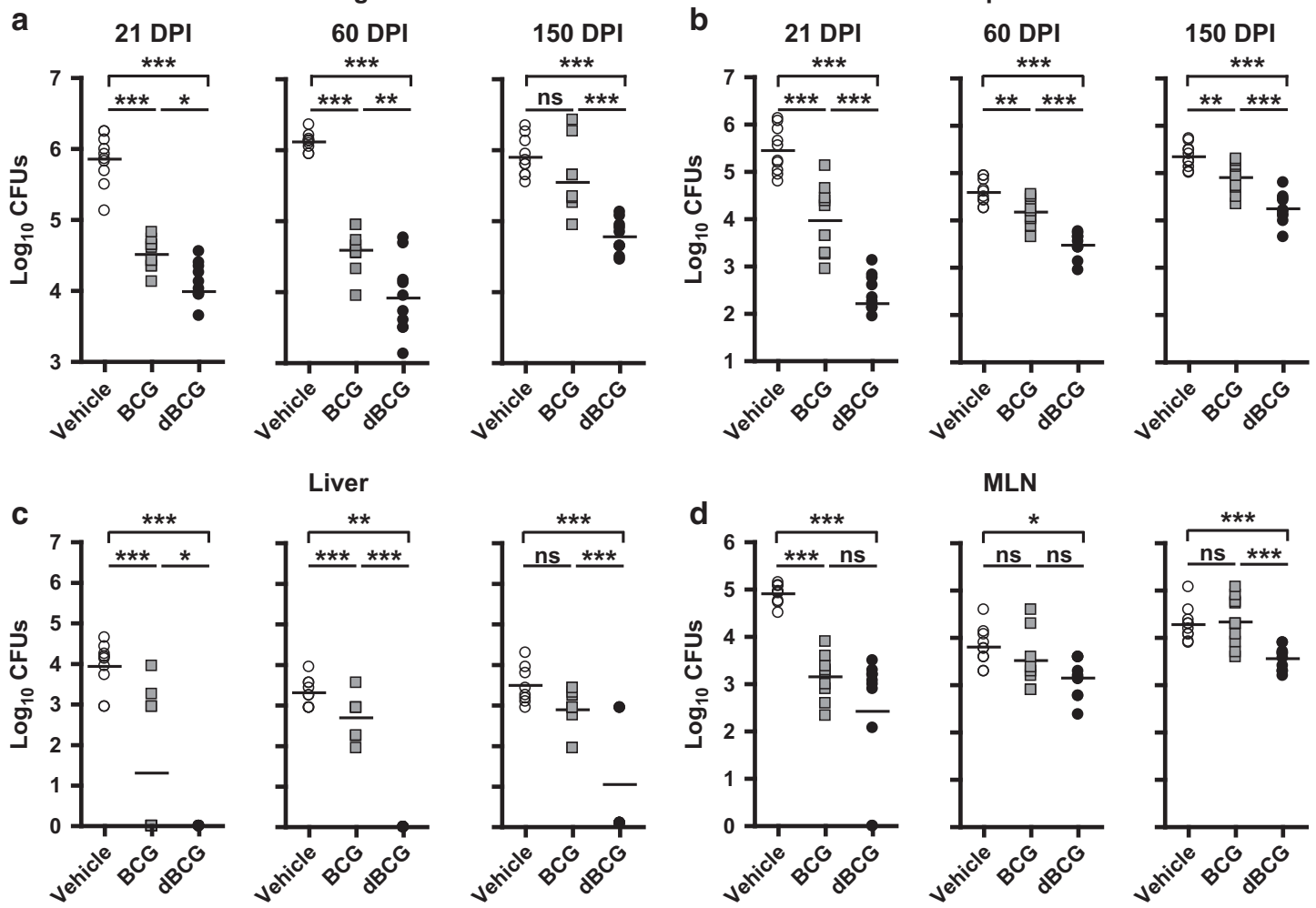

Fig. 4 Pulmonary vaccination with dBCG reduces M.tb bacterial burden in the lung and peripheral organs of infected mice. C57BL/6 mice were intranasally inoculated with PBS (vehicle; white circles) or $5 \times 10^{5}$ viable BCG (grey squares) or dBCG (black circles) bacilli. Fifty days later, mice were infected with a low dose aerosol of M.tb. At 21, 60, and 150 DPI mice were euthanized to assess bacterial burden in the (a) lung, (b) spleen, (c) liver, and (d) MLN. Colony counts reported are from experiments in which M.tb was grown in 7H11 agar supplemented with TCH which allows for the growth of M.tb, but not BCG. Pooled results from $n=2$ with 4-5 mice/group per time-point, mean \pm SEM; One-way ANOVA with Tukey's post hoc test, ${ }^{*} p<0.05,{ }^{* *} p<0.01,{ }^{* * *} p<0.001$; ns not significant, MLN mediastinal lymph node

Together, these data suggest that removal of non-polar lipids from BCG augments its efficacy as a vaccine against $M . t b$ by delaying its expansion.

Importantly, we determined if $\mathrm{dBCG}$ vaccination was able to reduce tissue damage by quantifying areas of cellular aggregation relative to the total size of the lung (Fig. 5). dBCG vaccination attenuated pulmonary inflammation at every time point studied. At 21 DPI small areas of cellular aggregation were visible in all three groups, but foci in the lung of vehicle-vaccinated and BCG mice were significantly larger compared to dBCG (Fig. 5a, b). Inflammatory foci doubled in size in the lung of vehicle-treated and BCG vaccinated mice at $60 \mathrm{DPI}$, whereas foci in the lung of dBCG-vaccinated mice remained small. We also observed significantly decreased pulmonary inflammation in the lung of dBCGvaccinated mice compared to BCG-vaccinated mice at this time point. At $150 \mathrm{DPI}$, the mean area of the inflammatory foci was $20.84 \pm 4.66 \%$ in vehicle-treated mice and $19.14 \pm 13.54 \%$ in BCGvaccinated mice, whereas in dBCG-vaccinated was $8.30 \pm 3.04 \%$. Importantly, the decrease in lung pathology was not due to a reduction in M.tb bacterial burden (Sup. Fig. S7A, B). Together the data suggest that $\mathrm{dBCG}$ is effective at reducing initial bacterial burden and at delaying pulmonary immunopathology.

Pulmonary vaccination with $\mathrm{dBCG}$ accelerates effector $\mathrm{T}$ cell responses in the lung upon challenge with M.tb

Next, we evaluated effector T cells responses in the lung of mice that had been vaccinated (DPV 50) or vaccinated and then challenged with M.tb (DPI 10 and 21) (Fig. 6a and Sup. Fig. S5C). We focused on the expression of $\mathrm{CD} 69$ on $\mathrm{CD}^{+}$and $\mathrm{CD}^{+}{ }^{+} \mathrm{T}$ cells, and the ability of T cells to produce IFNY or IL-17A. In BCG and $\mathrm{dBCG}$ groups, the number of resident $\mathrm{CD} 69$ expressing $C D 4^{+}$and
$\mathrm{CD}^{+} \mathrm{T}$ cells in the lung before M.tb challenge was increased relative to vehicle but did not differ between BCG and dBCG vaccinated mice. Infection with M.tb increased the number of $\mathrm{CD} 9^{+} \mathrm{CD}^{+}$and $\mathrm{CD} 69^{+} \mathrm{CD} 8^{+} \mathrm{T}$ cells in the lung of vehicle control mice, but the number of $\mathrm{CD} 69^{+} \mathrm{CD}^{+}$and $\mathrm{CD} 69^{+} \mathrm{CD} 8^{+} \mathrm{T}$ cells in the lung was enhanced further with $d B C G$ at 21 DPI (Fig. 6b). To further assess $T$ cell activation in the lung we evaluated two important effector mechanisms required for M.tb control, the production of IFNY and IL-17A by T cells. ${ }^{7,29-32}$ Although the number of $\mathrm{CD}^{+}{ }^{+} \mathrm{FNY}^{+}$and $\mathrm{CD}^{+} \mathrm{IFNy}^{+} \mathrm{T}$ cells in the lung was elevated 50 DPV in BCG and $\mathrm{ABCG}$ vaccinated mice, we did not observe statistically significant increases in the number of $\mathrm{CD}^{+}{ }^{+} \mathrm{FN}^{+}$and $\mathrm{CD} 8^{+} \mathrm{IFN} \gamma^{+}$T cells in the lung between BCG- or dBCG-vaccinated mice at any time point post M.tb infection (Fig. 6c). We did, however, observe significant increases in the number of $\mathrm{CD}^{+}{ }^{\mathrm{IL}}-17 \mathrm{~A}^{+}$and $\mathrm{CD} 8^{+} \mathrm{IL}-17 \mathrm{~A}^{+} \mathrm{T}$ cells in the lung (Fig. 6d), with significant increases in the number of $C D 8^{+} I L-17 A^{+}$ $T$ cells at $10 \mathrm{DPI}$ and significant increases in $\mathrm{CD} 4^{+} \mathrm{IL}-17 \mathrm{~A}^{+}$and $\mathrm{CD}^{+} \mathrm{IL}^{-17 \mathrm{~A}^{+}} \mathrm{T}$ cells at $21 \mathrm{DPI}$ in the lung of $\mathrm{dBCG}$-vaccinated mice.

Enhanced control of M.tb by dBCG is linked to IL-17A-associated responses during vaccination period

Having observed significant increases in the activation (CD69) and IL-17A, but not IFN $\gamma$, positive $T$ cells in the lung of $d B C G$ vaccinated mice infected with M.tb, we sought to evaluate whether these responses were antigen specific. Mice were vaccinated with $B C G$ or $\mathrm{dBCG}$, sacrificed at $50 \mathrm{DPV}$, and purified lung cells were cultured with a mixture of $M$.tb-derived antigens. Analysis of cell populations positive for CD69, IFNy, or IL-17A after stimulation revealed a decreasing trend for the total percentage of 
a

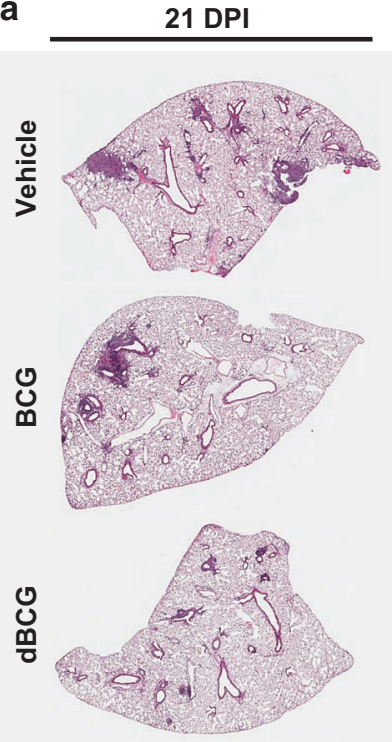

b

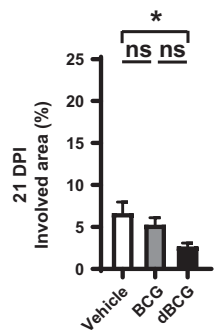

$60 \mathrm{DPI}$
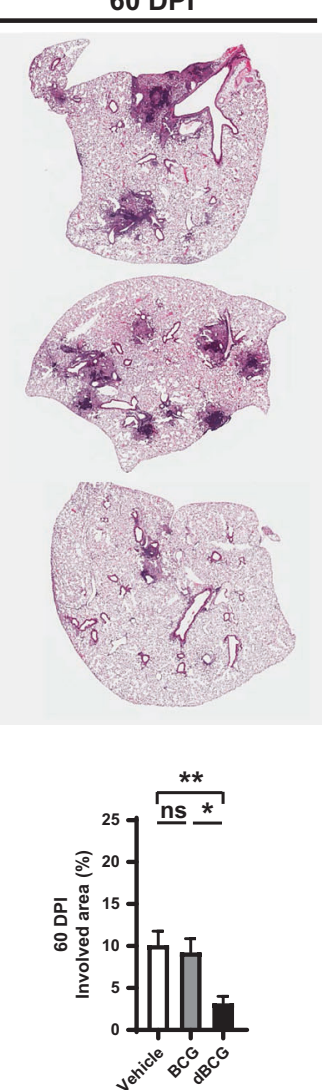

$150 \mathrm{DPI}$
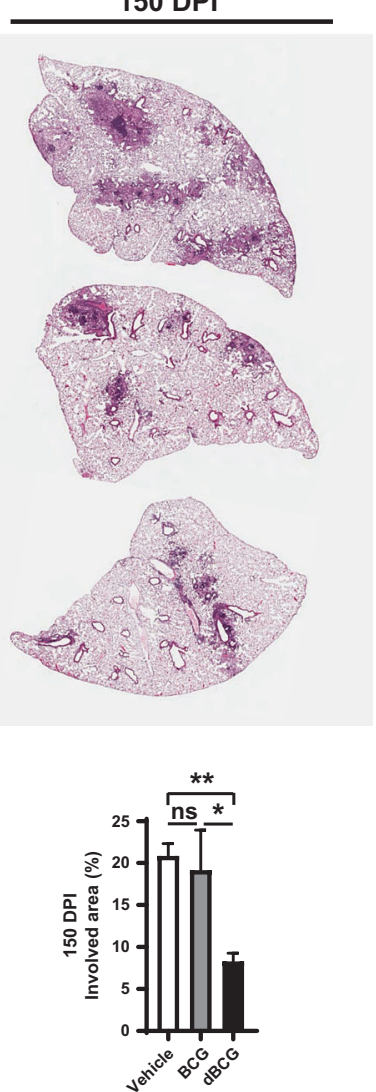

Fig. 5 Pulmonary vaccination with $\mathrm{dBCG}$ is associated with decreased M.tb lung pathology across time. C57BL/6 mice were intranasally inoculated with PBS (vehicle) or $5 \times 10^{5}$ viable BCG or dBCG bacilli. Fifty days later, mice were infected with a low dose aerosol of M.tb. a At 21 , 60 , and $150 \mathrm{DPI}$ mice were sacrificed, and lungs fixed in 10\% NBF, embedded in paraffin, sectioned, and stained with hematoxylin and eosin to visualize tissue morphology. b Areas of cell aggregation and infiltration (inflammation) in vehicle (white bars), BCG (grey bars) or dBCG (black bars) vaccinated mice were quantified using Aperio Imagescope by calculating the area of inflammatory foci (i.e., involvement) divided by the total area of the lung. Representative images at a final magnification of $\times 20$. Pooled results from $n=2$ with $4-5$ mice/group per time-point, mean \pm SEM; One-way ANOVA with Tukey's post-hoc test, ${ }^{*} p<0.05,{ }^{* *} p<0.01$; ns: not significant

IFN $\gamma$ positive cells in the $\mathrm{dBCG}$ group and an increasing trend for the number of IL-17A positive ones, in both the $\mathrm{CD}^{+}$and $\mathrm{CD} 8^{+}$ populations (Fig. 7a, Sup. Fig. S8). This led us to hypothesize that the induction of IL-17A-associated responses is important for dBCG-mediated immunity. To directly assess whether IL-17A contributes to the control of M.tb following $\mathrm{dBCG}$ vaccination, mice were vaccinated with BCG or dBCG and injected with anti-IL$17 \mathrm{~A}$ throughout the duration of the vaccination period beginning one day prior to vaccination, challenged with M.tb, and sacrificed at $21 \mathrm{DPI}$ to assess bacterial burden (Fig. 7b). As expected, naïve mice had the highest bacterial burden, and both vaccinations were able to significantly reduce bacterial burden in the lung. However, as previously observed, dBCG was superior to BCG at reducing M.tb bacterial burden. Neutralization of IL-17A had no significant effect on the bacterial burden in the BCG group, but its neutralization in the $\mathrm{dBCG}$ group abolished the increased protection associated with dBCG vaccination (Fig. 7c). Overall, our results suggest the non-polar lipid components of the mycobacterial cell wall negatively impact BCG's vaccine efficacy when delivered directly to the lungs, and directly implicates IL-17A as an important immune response modulator.

\section{DISCUSSION}

BCG remains the only vaccine available to prevent TB, yet it fails to confer long-term immunity against PTB. Despite its success against meningeal- and miliary-TB, BCG's efficacy against PTB must be improved if we wish to eradicate this disease. One underlying factor that may contribute to this phenomenon is the route of immunization with BCG, which does not reflect the route of natural infection with M.tb. M.tb has evolved to be a pulmonary pathogen by taking advantage of the immunoprivileged status of the lung, where inflammatory responses are tightly regulated to prevent excessive damaging inflammation. ${ }^{33}$ Vaccination with conventional BCG, on the other hand, is administered into the dermal layer of the skin. ${ }^{2}$ Multiple studies have suggested that BCG is more efficacious against M.tb when delivered directly into the lung..$^{5-7}$ Unfortunately, the presence of immunomodulatory and inflammatory lipids on the mycobacterial cell wall has restrained the use of BCG as a pulmonary vaccine in humans. Previous studies by Silva et al. ${ }^{11}$ and Indrigo et al. ${ }^{34}$ demonstrated the importance of PE extracted mycobacterial lipids, especially TDM, in the host-pathogen relationship that takes place in infections caused by mycobacteria in vitro and in vivo. As a result, we postulated that selective removal of inflammatory lipids from the cell wall of $\mathrm{BCG}^{11}$ would enable direct pulmonary vaccination with $B C G$.

Selective dBCG with PE effectively served as a more potent vaccine against $M$.tb when administered directly into the lungs. However, we acknowledge that $\mathrm{dBCG}$ still leads to increasing M.tb burden over time, but it does remain superior in its protection relative to conventional $B C G$, including reduced immunopathology. We thus suggest that by lowering initial lung inflammatory responses, we have identified an important improvement over 


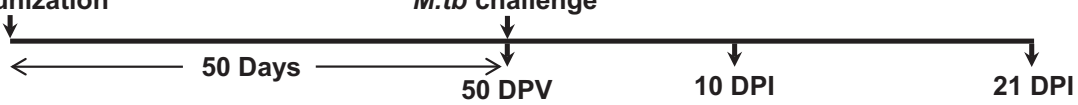

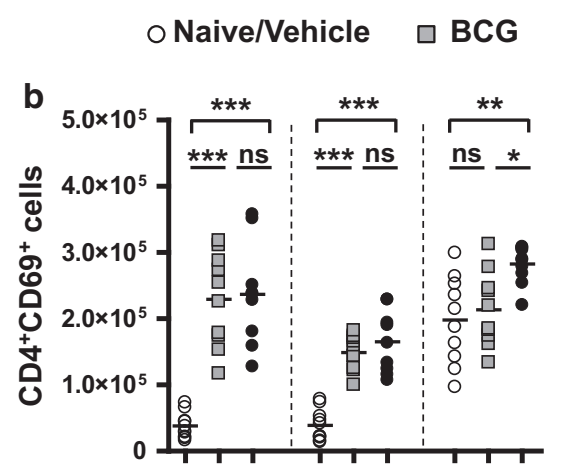

dBCG
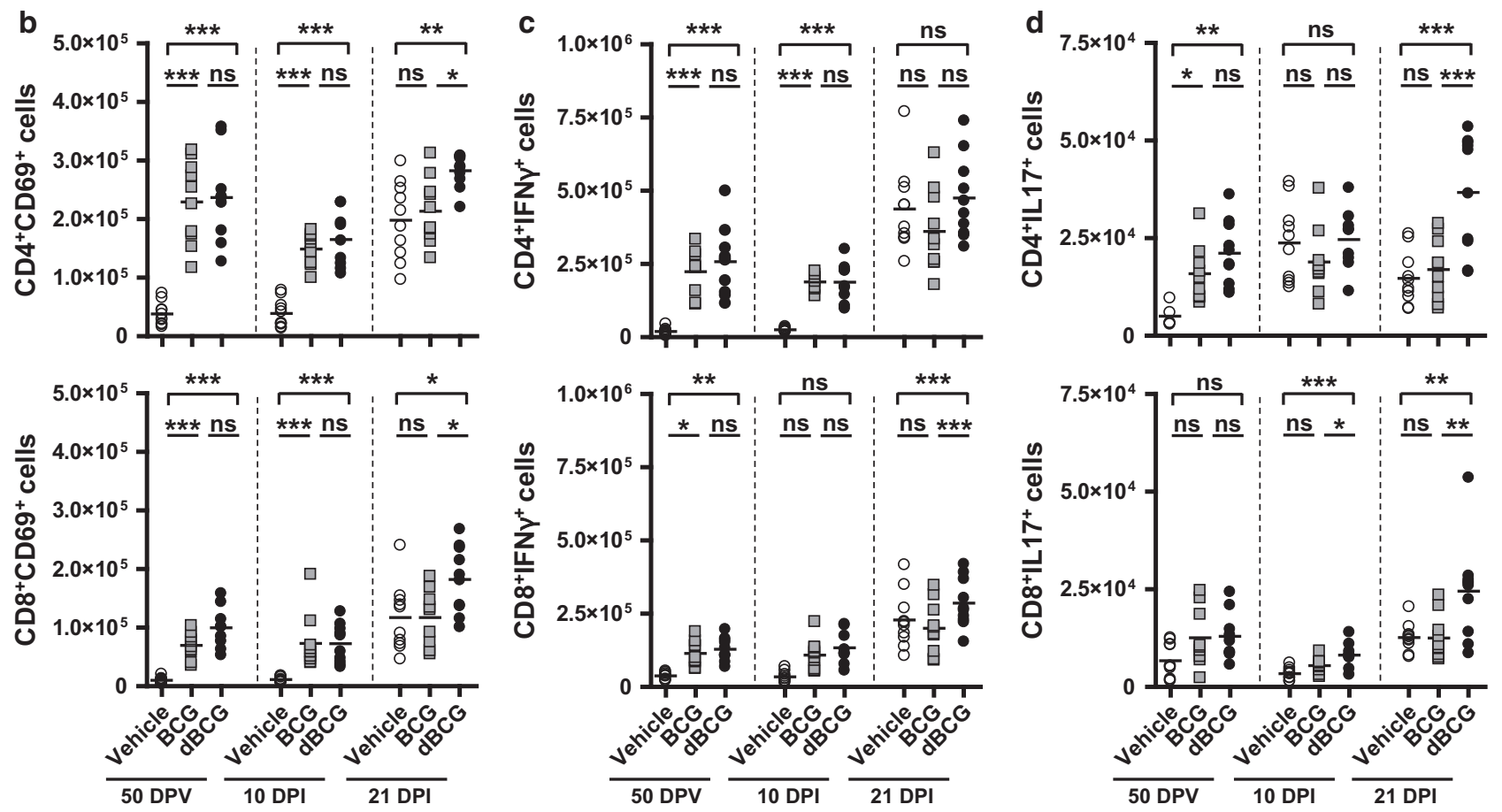

Fig. 6 Pulmonary vaccination with dBCG boosts CD69 and IL-17A, but not IFN $\gamma$, responses in the lung of M.tb infected mice. a Timeline showing experimental design. C57BL/6 mice were intranasally inoculated with vehicle (white circle) or $5 \times 10^{5}$ viable BCG (grey square) or dBCG (black diamond) bacilli. b T cell activation based on the expression of CD69 on CD4 ${ }^{+}$and CD8 ${ }^{+}$T cells at 50 DPV and at 10 and 21 DPI. CD69 was significantly increased in dBCG-vaccinated mice at $21 \mathrm{DPI}$. c Total number of CD4 ${ }^{+}$and CD8 ${ }^{+}$T cells staining positive for IFN $\gamma$ after stimulation with $\mathrm{CD} 3 / \mathrm{CD} 28$ in the presence of monensin. $\mathbf{d}$ Total number of $\mathrm{CD} 4^{+}$and $\mathrm{CD}^{+} \mathrm{T}$ cells staining positive for or IL-17A after stimulation with $\mathrm{CD} 3 / \mathrm{CD} 28$ in the presence of monensin. Mice vaccinated with $\mathrm{dBCG}$ had significantly higher proportions of $\mathrm{CD}^{+}{ }^{+} \mathrm{IL}-17 \mathrm{~A}^{+}$ cells (10 DPI) and CD4 ${ }^{+} \mathrm{IL}-17 \mathrm{~A}^{+}$and $\mathrm{CD} 8^{+} \mathrm{IL}-17 \mathrm{~A}^{+}(21 \mathrm{DPI})$ in the lung, while IFN $\gamma^{+}$cells were not significantly elevated in either vaccination group. Pooled experiment from $n=2$ with $4-5$ mice/group, mean \pm SEM; One-way ANOVA with Tukey's post-hoc test, ${ }^{*} p<0.05$, ${ }^{* *} p<0.01$, $* * * p<0.001$

conventional BCG. In this regard, pulmonary immunopathology is the primary reason why BCG has not been repurposed as a pulmonary vaccine ${ }^{8,9}$ and immunopathology of M.tb infected animal models is a major correlate of morbidity and mortality. ${ }^{2}$

Our data suggest that the improved protection and reduced pulmonary inflammation (to $\mathrm{dBCG}$ and M.tb) associated with $\mathrm{dBCG}$ vaccination is due to the removal of virulent lipids including TDM, MycB, PGL, TAGs, and PDIMs from the BCG cell wall, which can inhibit the development of effective immune responses and/or exacerbate inflammatory responses. In this context, TDM has been associated with the ability to inhibit fusion between phospholipid vesicles such as those required for the fusion of phagosomes with lysosomes. ${ }^{35}$ Phagosome-lysosome fusion is required for the killing of intracellular M.tb and for the subsequent presentation of foreign peptides along with MHC class II to T cells. ${ }^{36}$ TDM can also inhibit cellular energy metabolism, ${ }^{37}$ inhibit crosstalk between innate and adaptive immune cells, ${ }^{38}$ and induce apoptosis of lymphocytes. ${ }^{39}$ Some M.tb clinical isolates can synthesize the trisaccharide form of PGL, which can inhibit Toll-like receptor 2 (TLR2)-induced NF-KB activation, and thus the production of IL-1 $\beta$, TNF, and IL-6. ${ }^{17}$ Like PGL, the accumulation of TAGs on the mycobacterial cell surface can increase virulence. ${ }^{40}$ PDIMs also appear to play a role in the virulence of mycobacteria as suggested by M.tb strains that lack PDIM being less virulent in mouse models, ${ }^{20,41}$ a phenotype attributed to reduced binding of
M.tb to the plasma membrane of macrophages and increased phagosomal acidification. ${ }^{13}$ Other studies have linked the absence of PDIMs on M.tb to reduced survival of M.tb within macrophages, suggesting PDIMs protect $M . t b$ from early innate host responses. ${ }^{19}$ Thus, $d B C G$ has a significant impact on multiple virulence factors that influence both innate and adaptive immune elements.

We also anticipate that $\mathrm{dBCG}$ may begin rebuilding its cell wall soon after it enters an environment that is permissive to growth. However, mice vaccinated with $\mathrm{dBCG}$ had minimal pathology at $150 \mathrm{DPV}$ in the lung, indicating that in the case that dBCG can resynthesize the removed lipids, this potential reconstitution does not induce the inflammatory response associated with conventional BCG. Moreover once dBCG is inoculated, innate host cells may rapidly begin to kill the bacteria. This rapid killing, combined with a slow antigen presentation process, could explain why dBCG is more efficient at controlling M.tb growth. In this context, interestingly, at day 7, dBCG vaccination drove a transient decrease in nearly all innate cells studied. We attribute this decrease to the absence of inflammatory lipids on the dBCG cell wall, where $\mathrm{dBCG}$ could induce a transient cell turnover to exit the lung, or for the case of monocytes, it could accelerate their differentiation into lung macrophages (alveolar and/or interstitial) and/or dendritic cells. Conversely, the absence of immunomodulatory/inflammatory lipids in dBCG could also allow for the development of a stronger adaptive immune response by 
a


dBCG
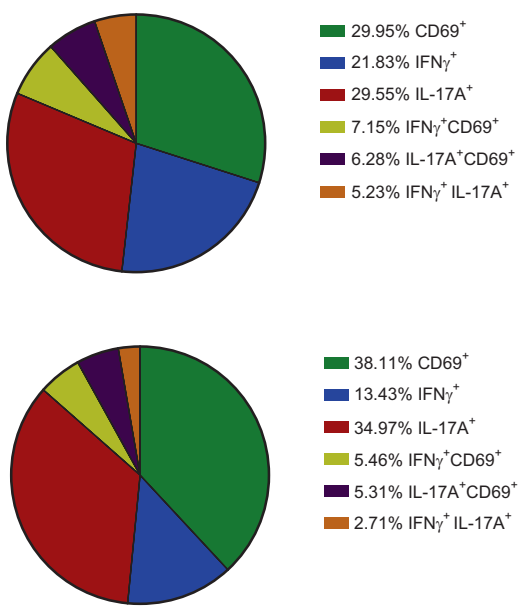

$38.11 \%{\mathrm{CD} 69^{+}}^{+}$ - $13.43 \% \mathrm{IFN}^{+}$ - $34.97 \% \mathrm{LL}-17 \mathrm{~A}^{+}$ $5.46 \%$ IFN $\gamma^{+} \mathrm{CD}^{+} 9^{+}$ - $5.31 \% \mathrm{IL}-17 \mathrm{~A}^{+} \mathrm{CD} 69^{+}$ $2.71 \% \mathrm{IFN} \gamma^{+} \mathrm{IL}-17 \mathrm{~A}^{+}$ b



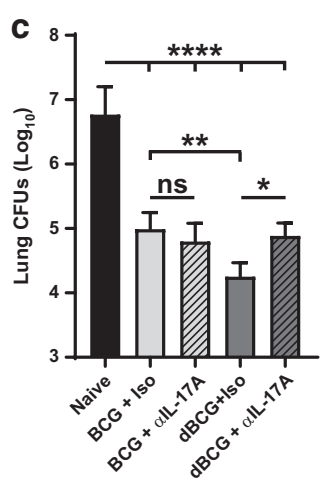

Fig. 7 IL-17A is an important mediator of the increased efficacy of dBCG against M.tb. a Purified lung cells of BCG or dBCG immunized mice sacrificed at 50 DPV were cultured with M.tb $\mathrm{H}_{37} \mathrm{R}_{\mathrm{v}}$ CFP $(10 \mu \mathrm{g})$ for $18 \mathrm{~h}$. The expression of CD69, IFN $\gamma$, and IL-17A was measured by flow cytometry. Each slice represents cells (CD4 or CD8) that express the corresponding molecule(s) as a percentage of the total number of cells analyzed. Overall, IFN $\gamma$ responses were less prominent in dBCG-immunized mice, while IL-17A responses were elevated. $\mathbf{b}$ Timeline depicting experimental design for IL-17A neutralization. C57BL/6 J mice were intranasally inoculated with vehicle (naïve) or $5 \times 10^{5}$ viable BCG or dBCG bacilli. IL-17A was depleted throughout the 50 day vaccination period beginning one day before vaccinations and was stopped three days prior to M.tb infection. c M.tb bacterial burden in the lung of naïve, BCG, or ABCG vaccinated mice injected with isotype or anti-IL-17A antibodies. Absence of vaccination (naive, black bar) results in the highest bacterial burden. Vaccination with BCG (light gray) reduced bacterial burden, while vaccination with dBCG (dark gray) reduced it even further. Depletion of IL-17A had no observable effect in the BCG group but ablated the additional protection associated with dBCG (dashed bars). Colony counts reported are from experiments in which $M$.tb was grown on 7H11 agar supplemented with TCH which allows for the growth of M.tb, but not BCG. Pooled results from $n=2$ with 4-5 mice/ group per time-point, mean \pm SEM; One-way ANOVA with Tukey's post hoc test, ${ }^{*} p<0.05,{ }^{* *} p<0.01$, ${ }^{* * * *} p<0.0001$, ns not significant. Note: Open line with multiple dashes compares the naïve group with all the other groups showing in all cases the same significance $(* * * * p<0.0001)$

bypassing the masking effect that the presence of these lipids on conventional BCG may have.

Pulmonary vaccination with $\mathrm{dBCG}$ may also accelerate the development of immunological memory and $T$ cell activation in the lung following M.tb infection. Although we observed a significant increase in the number of CD4 and CD8 T cells positive for CD69 in both BCG-vaccinated and ABCG-vaccinated groups at $50 \mathrm{DPV}$ and $10 \mathrm{DPI}, \mathrm{dBCG}$-vaccinated mice had higher numbers of $\mathrm{CD}^{+} \mathrm{CD}^{+} 9^{+}$and $\mathrm{CD}^{+} \mathrm{CD}^{+} 9^{+} \mathrm{T}$ cells in the lung $21 \mathrm{DPI}$, suggesting that vaccination with $\mathrm{dBCG}$ may accelerate $\mathrm{T}$ cell activation upon M.tb infection. We also assessed the number of $T$ cells capable of producing IFNY and IL-17A in the lung, important cytokines for M.tb containment. ${ }^{42,43}$ Unexpectedly, we did not observe any significant differences between BCGvaccinated and dBCG-vaccinated mice in the number of $T$ cells capable of producing IFNy prior to, or following, infection with $M$. $t b$ in the lung. Furthermore, the overall proportion of IFN $\gamma^{+} \mathrm{T}$ cells after stimulation with M.tb antigens was decreased in dBCGvaccinated mice at the time of infection. Since IFNy did not mediate the additional protection against M.tb associated with
dBCG vaccination, we assessed whether IL-17A was implicated. ${ }^{30,42}$ We observed significant increases in the number of CD8 ${ }^{+} I L-17 A^{+} T$ cells in the lung of $\mathrm{dBCG}$-vaccinated mice at 10 $\mathrm{DPI}$, and significant increases in the number of $\mathrm{CD} 4^{+} \mathrm{IL}-17 \mathrm{~A}^{+}$and $\mathrm{CD} 8^{+} \mathrm{IL}-17 \mathrm{~A}^{+}$cells in the lung at $21 \mathrm{DPI}$. Furthermore, the proportion of $\mathrm{T}$ cells positive for IL-17A after M.tb antigen stimulation was higher in dBCG-vaccinated mice. Depletion of IL-17A from dBCG-vaccinated mice during the vaccination period confirmed its requirement for controlling $M . t b$ bacterial burden in the lung. Thus, our data support that IFNY may have a limited ability to protect against M.tb in the lung and that IL-17A responses may be necessary for effective vaccination-induced mycobacterial immunity in the lung.

The key to this novel approach could be the rapid innate immune response that occurs after inoculation, which helps to develop strong adaptive immune responses. Although the pathology observed post-M.tb infection is likely due to M.tb, we cannot rule out that residual BCG is also responsible. We also stress that the significance of these findings lies in the route of immunization. It may be difficult to justify the use of conventional 
BCG as a pulmonary vaccine due to large amounts of inflammation generated in the lungs. However, dBCG could offer a solution; a less cytotoxic vaccine that could be inoculated directly into the lungs and that is associated with greater immunopathological protection and subsequent delayed M.tb expansion. Additionally, targeting IFN $\gamma$ responses may not necessarily increase immunity to mycobacteria in the lung. Instead, IL-17A responses may contribute significantly to mycobacterial immunity in the lung mucosa in the context of BCG vaccination.

Our data suggest that BCG cell wall lipids directly inhibit the generation of protective immune responses to M.tb. Whereas current vaccines typically fall under the categories of attenuated, inactivated, or subunit/conjugate, we have classified dBCG under a new category we named "selective attenuation". While the bacteria remain alive, and therefore capable of replenishing the extracted lipids on their cell walls, the removal of cell wall lipids significantly reduces its ability to survive within host cells. This allowed us to vaccinate via a more immunogenic route. Additionally, as high incidence of TB occurs in developing countries, particularly within rural communities that may lack access to routine health care, TB vaccine design should aim to develop single dose formulations. Vaccines that require administration of multiple components pose significant logistical challenges. For these reasons, our design was centered on developing a single-dose vaccine that can be safely delivered to the lung. We overcame both challenges via a simple lipid extraction using the readily available solvent PE. The procedure was fast, reproducible, inexpensive, and can be easily implemented into current vaccine production.

Further studies are required to determine the potential translation of our findings from mouse to humans. Logistically, dBCG may need to surpass the protective effects of conventional BCG in pre-clinical models. A ratio improvement of uninfected: latent infection:active TB disease in ABCG vaccinated NHPs could fast-track $d B C G$ for vaccine production due to the urgency of addressing the current TB epidemic. ${ }^{44}$ As $d B C G$ seems to cause less burden and delays immunopathology, this vaccine may allow for routes of vaccination previously considered too toxic (i.e., the lung), as well as potentially addressing the current safety concerns of using conventional BCG for immunocompromised individuals. Altogether our research highlights the complexities of the initial interaction between mycobacteria and the host and exemplifies that a thorough understanding of the mycobacterial cell wall can lead to a better understanding of the requirements for effective mycobacterial immunity.

\section{METHODS}

Ethics statement

All experimental procedures with animals were approved by the The Ohio State University IACUC (2012A00000132) and Texas Biomedical Research Institute IACUC (1611-MU). All mouse experiments were repeated at least twice $(n=2)$ with $4-5$ mice per group. For human subjects, this study was carried out in strict accordance with US Code of Federal and Local Regulations, Human Subjects IRB protocols: $2007 \mathrm{H} 0262$ and 2008H0119. Only healthy adult human subjects were recruited (50:50 F:M, 21-44 years old) with written informed consent.

Mice

Specific-pathogen-free C57BL/6 female mice (Jackson-Laboratories, Bar Harbor, ME) aged 6-8 weeks were hosted as described. $^{28,45}$ Mice were divided into three groups: Mockvaccinated (vehicle), PBS-treated-BCG-vaccinated (BCG), or PEtreated-BCG-vaccinated (dBCG). Each group contained 4-5 mice and experiments were repeated once for a total of 8-10 mice per group studied.
Growth conditions of mycobacteria and delipidation of $M$. bovis BCG strain Pasteur

GFP-M.tb Erdman (provided by Dr. Horwitz, UCLA, CA) ${ }^{46}$ and $M$. bovis BCG Pasteur strains [BCG, ATCC, \#35734] were grown as previously described. ${ }^{47}$ To delipidate BCG, freshly plated BCG was harvested between 9-14 days of growth into siliconated tubes (Fisher Scientific, Hampton, NH), suspended in $1 \mathrm{ml}$ of PE or PBS and vortexed for two minutes, rested for five minutes, and then pelleted at $6000 \times g$ for five minutes. The procedure was performed as previously described ${ }^{23,24}$ and repeated three times. The supernatants from the PE treated bacteria were collected, dried under $\mathrm{N}_{2}$, and kept at $-20^{\circ} \mathrm{C}$ until further analysis. Treated bacteria used for in vitro or in vivo studies were dried briefly in the biosafety cabinet to evaporate the excess solvent, washed with PBS, and suspended in PBS prior to use. The viability of BCG and dBCG was determined counting colonies three to four weeks later. ${ }^{47}$ Generation of a viable single cell suspension of BCG after delipidation was confirmed by staining with a live/dead solution containing propidium iodide $(535 \mathrm{~nm})$ and SYTO $^{\circ}(485 \mathrm{~nm})$ for ten minutes (ThermoFisher Scientific Cat. L7012). Bacteria were visualized under an Olympus FluoView FV10i confocal microscope at a final magnification of $\times 600$ with a $\times 3$ zoom.

\section{Analysis of the extracted lipids}

To assess the remaining lipids on the cell wall of BCG post PE extraction, PE-treated bacteria were further extracted with C:M $(2: 1, v / v)$ at $37^{\circ} \mathrm{C}$ for $12 \mathrm{~h}$. PE and C:M extracted lipids (spotted 100 $\mu \mathrm{g}$ per lane) were analyzed by TLC using aluminum-backed TLC plates with the following solvent systems: For TDM, MycB, and PGL (C:M 95:5, v/v); for PDIMs and TAGs (PE:acetone, 96:4, v/v); and PIMs (C:acetic acid:M:water, 40:25:3:6, v/v/v/v). TLC bands were visualized by first spraying the plates with $10 \% \mathrm{H}_{2} \mathrm{SO}_{4}$ in $\mathrm{H}_{2} \mathrm{O}$ followed by charring at $100^{\circ} \mathrm{C}$. Developed TLCs were imaged using an Epson Perfection V600 photo scanner. Quantification was performed using the NIH software ImageJ. Total BCG extracts were obtained by sequential extractions using C:M $(2: 1, v / v) ; C: M(1: 2, v /$ v) and C:M:water (10:10:3, v/v/v) as we previously reported. ${ }^{48}$

\section{Vaccination and M.tb aerosol infection}

C57BL/6 mice were anesthetized with an aerosolized solution containing $2-5 \%$ isoflurane. A single cell suspension of BCG or dBCG containing $\sim 5 \times 10^{5}$ viable bacilli in $50 \mu$ l was intranasally administrated evenly between the two nares allowing for inhalation into the lungs one mouse at a time. Following administration, mice were held in an upright position for $15 \mathrm{~s}$ to ensure the entire inoculum was inhaled. For M.tb studies, mice were infected via aerosol with a low dose of M.tb using the GlasCol (Terre Haute, IN) inhalation exposure system as we described. ${ }^{28,45}$

\section{Histopathology}

Lung tissue sections were prepared, processed and analyzed as described by a board-certified veterinarian in a blinded manner. ${ }^{45}$

In vitro infection of human macrophages

In vitro infections were performed as described previously. For specific details, see supplementary materials.

Lung cell isolation

Lung cells were isolated as previously described. ${ }^{49}$ Cell suspensions were counted using Trypan blue to exclude dead cells and resuspended at a working concentration in c-DMEM or fixed for flow cytometry.

Immunophenotyping by flow cytometry Lung cells were prepared for flow cytometry as described previously. ${ }^{45}$ For specific details, see supplementary materials. 
Neutralization of IL-17A

IL-17A (500 $\mu$ g; Clone: 17F3; BioXCell, West Lebanon, NH) depletion antibody or whole mouse IgG1, к (500 $\mu$; Clone: MOPC-21; BioXCell) isotype control were administered in $100 \mu \mathrm{l}$ into the intraperitoneal cavity twelve times over a period of 50 days beginning one day prior to vaccination and ending three days prior to M.tb challenge.

Statistical analysis

Statistical significance was determined using Prism 7 software (GraphPad). The unpaired, two-tailed Student's $t$-test was used for two group comparisons. Multiple comparisons were analyzed using one-way ANOVA with the Holm-Sidak's multiple comparison test to compare all groups against control (naïve) $(*)$, groups within the same time points $(\sharp)$, and groups across different time points $\left({ }^{\S}\right)$. Statistical significance was reported as ${ }^{*}, \mathbb{\S}, \sharp p<0.05$;

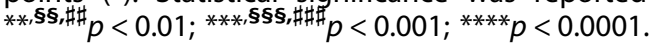

Supplementary information is linked to the online version of the paper at http://www.nature.com/mi. Additional methods on in vitro infection of human macrophages, SDS-PAGE and wholecell ELISA for ManLAM, bacterial burden enumeration, cytokine/ $\mathrm{LDH}$ quantification by ELISA, and flow cytometry reagents available in supplementary materials.

\section{ACKNOWLEDGEMENTS}

This work was supported by the National Institute of Allergy and Infectious Diseases grant numbers [AI073856 and Al093570] to J.B.T.; by a National Institute on Aging grant number [AG051428] supporting J.T. and J.B.T.; and by The Ohio State University (OSU) College of Medicine Systems in Integrative Biology Training Grant [T32-GM068412], and NIH/NIAID [AI039570-S1] and NIH/NIA [AG051428-S1] supplements supporting J.I.M. This study was also partially supported by a CTSA award UL1TR001070 from the National Center for Advancing Translational Sciences. Its contents are solely the responsibility of the authors and do not necessarily represent official views of the National Center for Advancing Translational Sciences or the National Institutes of Health. We thank the University Laboratory Animal Resources facility and personnel, the Biosafety Level 3 program facility and personnel, and the Comparative Pathology and Mouse Phenotyping Shared Resource (P30 CA016058) at OSU for their technical support.

\section{AUTHOR CONTRIBUTIONS}

Conceptualization, J.I.M., J.T., and J.B.T.; Methodology, J.I.M., J.T., and J.B.T.; Investigation, J.I.M., A.P.H., S.S., C.H.C., V.D., and G.B.; Writing - Original Draft, J.I.M.; Writing - Review \& Editing, J.I.M., J.T., and J.B.T.; Funding Acquisition, J.T and J.B.T.; Supervision, J.T. and J.B.T.

\section{ADDITIONAL INFORMATION}

The online version of this article (https://doi.org/10.1038/s41385-019-0148-2) contains supplementary material, which is available to authorized users.

Competing interests: The authors declare no competing interests.

Publisher's note: Springer Nature remains neutral with regard to jurisdictional claims in published maps and institutional affiliations.

\section{REFERENCES}

1. WHO. Global Tuberculosis Report 2018. pp. 1-277. (WHO Organization, Geneva, 2018). http://apps.who.int/iris/bitstream/handle/10665/274453/9789241565646eng.pdf?ua $=1$.

2. Moliva, J. I., Turner, J. \& Torrelles, J. B. Prospects in Mycobacterium bovis Bacille Calmette et Guerin (BCG) vaccine diversity and delivery: why does BCG fail to protect against tuberculosis? Vaccine 33, 5035-5041 (2015).

3. Moliva, J. I., Turner, J. \& Torrelles, J. B. Immune responses to BCG vaccination: why do they fail to protect against Mycobacterium tuberculosis? Front Immunol. 8, 407 (2017).
4. Lambert, P. H. \& Laurent, P. E. Intradermal vaccine delivery: will new delivery systems transform vaccine administration? Vaccine 26, 3197-3208 (2008).

5. Derrick, S. C., Kolibab, K., Yang, A. \& Morris, S. L. Intranasal administration of Mycobacterium bovis BCG induces superior protection against aerosol infection with Mycobacterium tuberculosis in mice. Clin. Vaccin. Immunol. 21, 1443-1451 (2014).

6. Perdomo, C. et al. Mucosal BCG vaccination induces protective lung-resident memory T cell populations against tuberculosis. MBio 7, 10.1128/mBio.01686-16 (2016).

7. Aguilo, N. et al. Pulmonary but not subcutaneous delivery of BCG vaccine confers protection to tuberculosis-susceptible mice by an Interleukin 17-dependent mechanism. J. Infect. Dis. 213, 831-839 (2016).

8. Tree, J. A. et al. Intranasal bacille Calmette-Guerin (BCG) vaccine dosage needs balancing between protection and lung pathology. Clin. Exp. Immunol. 138, 405-409 (2004).

9. Nuermberger, E. L., Yoshimatsu, T., Tyagi, S., Bishai, W. R. \& Grosset, J. H. Paucibacillary tuberculosis in mice after prior aerosol immunization with Mycobacterium bovis BCG. Infect. Immun. 72, 1065-1071 (2004).

10. Geisel, R. E., Sakamoto, K., Russell, D. G. \& Rhoades, E. R. In vivo activity of released cell wall lipids of Mycobacterium bovis bacillus Calmette-Guerin is due principally to trehalose mycolates. J. Immunol. 174, 5007-5015 (2005).

11. Silva, C. L., Ekizlerian, S. M. \& Fazioli, R. A. Role of cord factor in the modulation of infection caused by mycobacteria. Am. J. Pathol. 118, 238-247 (1985).

12. Chauhan, P. et al. Secretory phosphatases deficient mutant of Mycobacterium tuberculosis imparts protection at the primary site of infection in guinea pigs. PLOS. ONE 8, e77930 (2013).

13. Astarie-Dequeker, C. et al. Phthiocerol dimycocerosates of $M$. tuberculosis participate in macrophage invasion by inducing changes in the organization of plasma membrane lipids. PLoS. Pathog. 5, e1000289 (2009).

14. Sinsimer, D. et al. The phenolic glycolipid of Mycobacterium tuberculosis differentially modulates the early host cytokine response but does not in itself confer hypervirulence. Infect. Immun. 76, 3027-3036 (2008).

15. Cambier, C. J., O'Leary, S. M., O'Sullivan, M. P., Keane, J. \& Ramakrishnan, L. Phenolic glycolipid facilitates mycobacterial escape from microbicidal tissueresident macrophages. Immunity 47, 552-565 e554 (2017).

16. Reed, M. B. et al. A glycolipid of hypervirulent tuberculosis strains that inhibits the innate immune response. Nature 431, 84-87 (2004).

17. Arbues, A., Lugo-Villarino, G., Neyrolles, O., Guilhot, C. \& Astarie-Dequeker, C. Playing hide-and-seek with host macrophages through the use of mycobacterial cell envelope phthiocerol dimycocerosates and phenolic glycolipids. Front Cell Infect. Microbiol. 4, 173 (2014).

18. Arbues, A. et al. Trisaccharides of phenolic glycolipids confer advantages to pathogenic mycobacteria through manipulation of host-cell pattern-recognition receptors. ACS Chem. Biol. 11, 2865-2875 (2016).

19. Day, T. A. et al. Mycobacterium tuberculosis strains lacking surface lipid phthiocerol dimycocerosate are susceptible to killing by an early innate host response. Infect. Immun. 82, 5214-5222 (2014).

20. Cox, J. S., Chen, B., McNeil, M. \& Jacobs, W. R. Jr. Complex lipid determines tissuespecific replication of Mycobacterium tuberculosis in mice. Nature 402, 79-83 (1999).

21. Tran, V., Ahn, S. K., Ng, M., Li, M. \& Liu, J. Loss of lipid virulence factors reduces the efficacy of the BCGvaccine. Sci. Rep. 6, 29076 (2016).

22. Indrigo, J., Hunter, R. L. Jr. \& Actor, J. K. Cord factor trehalose 6,6'-dimycolate (TDM) mediates trafficking events during mycobacterial infection of murine macrophages. Microbiology 149, 2049-2059 (2003).

23. Bloch, H. Studies on the virulence of tubercle bacilli: isolation and biological properties of a constituent of virulent organisms. J. Exp. Med 91, 197-219 (1950).

24. Noll, H., Bloch, H., Asselineau, J. \& Lederer, E. The chemical structure of the cord factor of Mycobacterium tuberculosis. Biochim Biophys. Acta 20, 299-309 (1956).

25. Smith, K. C., Orme, I. M. \& Starke, J. R. Tuberculosis vaccines. In Vaccines $6 \mathrm{e}$, Plotkin, Offit \& Orenstein (Eds), Philadelphia: WB Saunders, 35, 789-811 (2013).

26. Vogelzang, A. et al. Central memory $C D 4+T$ cells are responsible for the recombinant Bacillus Calmette-Guerin DeltaureC::hly vaccine's superior protection against tuberculosis. J. Infect. Dis., 10.1093/infdis/jiu347 (2014).

27. Mueller, S. N., Gebhardt, T., Carbone, F. R. \& Heath, W. R. Memory T cell subsets, migration patterns, and tissue residence. Annu. Rev. Immunol. 31, 137-161 (2013).

28. Cyktor, J. C., Carruthers, B., Beamer, G. L. \& Turner, J. Clonal Expansions of CD8(+) T Cells with IL-10 secreting capacity occur during chronic Mycobacterium tuberculosis infection. PLOS. ONE 8, e58612 (2013).

29. Flynn, J. L. et al. An essential role for interferon gamma in resistance to Mycobacterium tuberculosis infection. J. Exp. Med 178, 2249-2254 (1993).

30. Khader, S. A. et al. IL-23 and IL-17 in the establishment of protective pulmonary $\mathrm{CD} 4+\mathrm{T}$ cell responses after vaccination and during Mycobacterium tuberculosis challenge. Nat. Immunol. 8, 369-377 (2007). 
31. Effros, R. B. \& Walford, R. L. Diminished T-cell response to influenza virus in aged mice. Immunology 49, 387-392 (1983).

32. Cooper, A. M. et al. Disseminated tuberculosis in interferon gamma genedisrupted mice. J. Exp. Med. 178, 2243-2247 (1993).

33. Cooper, A. M. Cell-mediated immune responses in tuberculosis. Annu. Rev. Immunol. 27, 393-422 (2009).

34. Indrigo, J., Hunter, R. L. Jr. \& Actor, J. K. Influence of trehalose 6,6'-dimycolate (TDM) during mycobacterial infection of bone marrow macrophages. Microbiology 148, 1991-1998 (2002).

35. Spargo, B. J., Crowe, L. M., loneda, T., Beaman, B. L. \& Crowe, J. H. Cord factor $(a, a-$ trehalose $6,6^{\prime}$-dimycolate) inhibits fusion between phospholipid vesicles. Proc. Natl Acad. Sci. USA 88, 737-740 (1991).

36. Pieters, J. Mycobacterium tuberculosis and the macrophage: Maintaining a balance. Cell Host. Microbe 3, 399-407 (2008).

37. Fox, C. J., Hammerman, P. S. \& Thompson, C. B. Fuel feeds function: energy metabolism and the T-cell response. Nat. Rev. Immunol. 5, 844-852 (2005).

38. Kan-Sutton, C., Jagannath, C. \& Hunter, R. L. Jr. Trehalose 6,6'-dimycolate on the surface of Mycobacterium tuberculosis modulates surface marker expression for antigen presentation and costimulation in murine macrophages. Microbes Infect. 11, 40-48 (2009).

39. Ozeki, Y. et al. In vivo induction of apoptosis in the thymus by administration of mycobacterial cord factor (trehalose 6,6'-dimycolate). Infect. Immun. 65, 1793-1799 (1997).

40. Reed, M. B., Gagneux, S., Deriemer, K., Small, P. M. \& Barry, C. E. III The W-Beijing lineage of Mycobacterium tuberculosis overproduces triglycerides and has the DosR dormancy regulon constitutively upregulated. J. Bacteriol. 189, 2583-2589 (2007).
41. Cox, D., Tseng, C. C., Bjekic, G. \& Greenberg, S. A. A requirement for phosphatidylinositol 3-kinase in pseudopod extension. J. Biol. Chem. 274, 1240-1247 (2000).

42. Gopal, R. et al. Unexpected role for IL-17 in protective immunity against hypervirulent Mycobacterium tuberculosis HN878 infection. PLoS. Pathog. 10, e1004099 (2014).

43. Pitt, J. M. et al. Blockade of IL-10 signaling during bacillus Calmette-Guerin vaccination enhances and sustains Th1, Th17, and innate lymphoid IFN-gamma and IL-17 responses and increases protection to Mycobacterium tuberculosisinfection. J. Immunol. 189, 4079-4087 (2012).

44. Fauci, A. S. Addressing the tuberculosis epidemic: 21 st century research for an ancient disease. JAMA 320, 1315-1316 (2018).

45. Moliva, J. I. et al. Exposure to human alveolar lining fluid enhances Mycobacterium bovis BCG vaccine efficacy against Mycobacterium tuberculosis infection in a CD8 +T-cell-dependent manner. Mucosal Immunol., https://doi.org/10.1038/ mi.2017.80 (2017).

46. Tullius, M. V., Harth, G. \& Horwitz, M. A. High extracellular levels of Mycobacterium tuberculosis glutamine synthetase and superoxide dismutase in actively growing cultures are due to high expression and extracellular stability rather than to a protein-specific export mechanism. Infect. Immun. 69, 6348-6363 (2001).

47. Arcos, J. et al. Human lung hydrolases delineate Mycobacterium tuberculosismacrophage interactions and the capacity to control infection. J. Immunol. 187, 372-381 (2011).

48. Torrelles, J. B. et al. Identification of Mycobacterium tuberculosis clinical isolates with altered phagocytosis by human macrophages due to a truncated lipoarabinomannan. J. Biol. Chem. 283, 31417-31428 (2008).

49. Canan, C. H. et al. Characterization of lung inflammation and its impact on macrophage function in aging. J. Leukoc. Biol. 96, 473-480 (2014). 